in vivo $33: 1485-1492(2019)$

doi:10.21873/invivo.11628

\title{
Targeted Sequencing of Tubo-ovarian and Peritoneal High-grade Serous Carcinoma With Wild-type p53 Immunostaining Pattern
}

\author{
HAN-NA KIM ${ }^{1}$, HA YOUNG WOO ${ }^{2}$, SUNG-IM DO ${ }^{3}$ and HYUN-SOO KIM ${ }^{1}$ \\ ${ }^{1}$ Department of Pathology and Translational Genomics, Samsung Medical Center, \\ Sungkyunkwan University School of Medicine, Seoul, Republic of Korea; \\ ${ }^{2}$ Department of Pathology, Severance Hospital, Yonsei University College of Medicine, Seoul, Republic of Korea; \\ ${ }^{3}$ Department of Pathology, Kangbuk Samsung Hospital, \\ Sungkyunkwan University School of Medicine, Seoul, Republic of Korea
}

\begin{abstract}
Background/Aim: We aimed to demonstrate the use of next-generation sequencing (NGS) to confirm the presence of tumor protein 53 (TP53) mutations in tuboovarian and peritoneal high-grade serous carcinoma (HGSC) with a wild-type p53 immunostaining pattern and investigate whether the TP53 mutational status is altered by chemotherapy. Materials and Methods: A commercial NGS panel comprising 171 genes was used to analyze the genetic profiles of 15 HGSC samples. Paired specimens obtained before and after chemotherapy were available for four patients. Results: All examined samples exhibited TP53 mutations. For all the patients who underwent neoadjuvant or postoperative adjuvant chemotherapy, TP53 mutations identified in samples obtained after chemotherapy were the same as those detected in pre-chemotherapeutic samples. Conclusion: HGSCs exhibit TP53 mutations even though a subset of HGSCs displayed a wild-type p53 immunostaining pattern. Chemotherapy does not affect the TP53 mutational status in HGSC.
\end{abstract}

This article is freely accessible online.

Correspondence to: Hyun-Soo Kim, Department of Pathology and Translational Genomics, Samsung Medical Center, Sungkyunkwan University School of Medicine, 81, Irwon-ro, Gangnam-gu, Seoul 06351, Republic of Korea. Tel: +82 234101243, Fax: +82 234100025, e-mail: hyun-soo.kim@samsung.com; Sung-Im Do, Department of Pathology, Kangbuk Samsung Hospital, Sungkyunkwan University School of Medicine, 29, Saemunan-ro, Jongno-gu, Seoul 03181, Republic of Korea. Tel: +82 220012393, Fax: +82 220012398, e-mail: sungim.do@samsung.com

Key Words: Ovary, high-grade serous carcinoma, p53, immunohistochemistry, TP53, next-generation sequencing.
Tumor protein 53 (TP53) is the most frequently mutated gene in malignancies. TP53 encodes for a transcription factor, p53, that initiates the transcription of genes involved in cell cycle arrest, cellular senescence, apoptosis, metabolism, DNA repair, and other processes following cellular stress $(1,2)$. p53, a critical tumor suppressor, plays a fundamental and multifaceted role in the development and progression of malignancies (3). In the absence of cellular stress, wild-type p53 is maintained at low levels, while in response to cellular stress, p53 is stabilized and activated through numerous mechanisms $(4,5)$. Activated wild-type p53 promotes processes consistent with tumor suppression, whereas mutation of p53 results in the loss of these tumorsuppressive functions.

Wild-type p53 is relatively unstable and has a short halflife, which makes it undetectable by immunostaining $(6,7)$. In contrast, mutant p53 has a much longer half-life and accumulates in the nucleus, thereby is detected by immunohistochemistry (7). TP53 mutations include singlebase substitutions leading to missense or non-sense point mutations, in-frame deletions or insertions, frameshift deletions or insertions, as well as mutations that affect splicing sites (5). Diffuse and strong nuclear p53 expression is regarded as indicative of a missense TP53 mutation $(8,9)$, and the complete absence of p53 immunoreactivity results from a nonsense TP53 mutation, leading to the formation of a truncated, non-immunoreactive protein (10-12).

Tubo-ovarian and peritoneal high-grade serous carcinoma (HGSC) is characterized by high frequency of pathogenic TP53 mutations. HGSC is the eighth-most frequent cause of cancer-related deaths in women worldwide (13). Most ovarian carcinoma cases are diagnosed at advanced stages, at which point, the five-year survival rate is approximately $25 \%(5,14)$. The existing therapeutic options for patients with tubo-ovarian and peritoneal HGSC are limited to 
aggressive debulking surgery and postoperative platinumbased adjuvant chemotherapy. An increased understanding of the alterations in the expression of genes and proteins involved in ovarian carcinogenesis may aid in improving the diagnosis and treatment of HGSC. A diagnostic or prognostic biomarker for ovarian carcinoma is, thus, urgently needed to guide the treatment of these patients.

It has recently been suggested that all HGSC cases are, in fact, TP53-mutants (15). This finding is in contrast to that previously known from studies that used less sensitive methods such as direct sequencing and focused on hot-spot regions of this gene only $(7,16,17)$. A precise validation of this practice by comparison with detailed sequencing data has been limited. We recently reported the direct sequencing results of TP53 in a cohort of HGSC in parallel with p53 immunostaining results (9). In this study, next-generation sequencing (NGS) was used to confirm the data previously obtained and to investigate whether the TP53 mutational status is altered by preoperative neoadjuvant or postoperative adjuvant chemotherapy in a larger cohort of HGSC with wild-type p53 immunostaining pattern.

\section{Materials and Methods}

Case selection. Following approval (4-2017-0993) by the Institutional Review Board, 240 cases of tubo-ovarian and peritoneal HGSC were initially selected from the archives of the Department of Pathology at the Severance Hospital (Seoul, Republic of Korea). We extracted 11 HGSC cases showing wild-type p53 immunostaining pattern. Clinical and pathological information, including the age of patient at initial diagnosis, tumor location, histological grade, International Federation of Gynecology and Obstetrics (FIGO) stage, type of surgical treatment, and addition of neoadjuvant or postoperative adjuvant chemotherapy, was obtained from the electronic medical record system and pathology reports. Two patients whose tumors showed mutant p53 immunostaining pattern (as a positive control) and one patient who underwent hysterectomy with left salpingo-oophorectomy for uterine leiomyoma (as a negative control) were also included.

Pathological examination. The resected tissues were initially examined by two pathologists, followed by fixation in $10 \%$ neutral-buffered formalin for 12-24 h. The tissues were then examined macroscopically and sectioned. After processing with an automatic tissue processor (Peloris II, Leica Microsystems, Newcastle Upon Tyne, UK), the sections were embedded in paraffin blocks. Four-micrometer-thick slices were sectioned from each formalin-fixed, paraffin-embedded (FFPE) tissue block using a rotary microtome (RM2245, Leica Microsystems) and stained with hematoxylin and eosin using an automatic staining instrument (Ventana Symphony System, Ventana Medical Systems, Tucson, AZ, USA). After staining, the slides were covered with a glass coverslip and sent to a board-certified pathologist specialized in gynecological oncology. The pathologist examined the hematoxylin and eosin-stained slides by light microscopy (BX43 System Microscope, Olympus, Tokyo, Japan) and made pathological diagnoses. In addition, the most representative slide for each case was chosen for subsequent immunostaining and sequencing.
Immunohistochemical staining. Immunostaining was performed using an automatic instrument [Ventana Benchmark XT (Ventana Medical Systems)] according to the manufacturer's recommendations $(9,18-35)$. Antigen retrieval was performed using Cell Conditioning Solution (CC1, Ventana Medical Systems). The 4- $\mu$ m-thick, formalin-fixed, paraffin-embedded sections were incubated with anti-p53 antibody (1:300, clone DO-7, Novocastra, Newcastle Upon Tyne, UK). After chromogenic visualization using an ultraView Universal DAB Detection Kit (Ventana Medical Systems), sections were counterstained with hematoxylin. Appropriate positive and negative controls were concurrently stained to validate the staining method. Negative control was prepared by substituting non-immune serum for primary antibody, which resulted in no detectable staining. The p53 immunostaining pattern was interpreted as a missense mutation, nonsense mutation, or wild-type pattern when p53 expression was diffuse and strong (>60\% of tumor cell nuclei), completely absent $(0 \%)$, or focal and weakly positive, respectively $(9,19,20,23)$.

Targeted sequencing. Genomic DNA was extracted using a QIAamp DNA FFPE Tissue Kit (Qiagen, Valencia, CA, USA). Coding exons and the flanking regions of 171 genes (Table I) were enriched using the SureSelectXT Reagent Kit (Agilent Technologies, Santa Clara, CA, USA) $(19,23)$. The products were sequenced on a HiSeq 2500 System (Illumina, San Diego, CA, USA) using paired-end reads. The reads were aligned to a reference genome sequence (Genome Reference Consortium Human Build 37) obtained from the University of California Santa Cruz Genome Browser database (https://genome.ucsc.edu/) (36), and duplicate reads were removed. Burrows-Wheeler Aligner (http://biobwa.sourceforge.net/), SAMtools (http://samtools.sourceforge.net/), Picard Tools (http://broadinstitute.github.io/picard/), and Genome Analysis Toolkit (https://software.broadinstitute.org/gatk/) were used for sorting Sequence Alignment Map/Binary Alignment Map files, duplicate marking, and local realignment, respectively. Local realignment and base recalibration were performed using the Single Nucleotide Polymorphism Database (https://www.ncbi.nlm.nih.gov/ projects/SNP/), Mills indel reference (37), HapMap (https:// www.ncbi.nlm.nih.gov/probe/docs/projhapmap/), and Omni (http:// www.internationalgenome.org/category/omni/). Single nucleotide variants, insertions, and deletions were identified using the MuTect (http://archive.broadinstitute.org/cancer/cga/mutect) and Pindel (http://gmt.genome.wustl.edu/packages/pindel/), respectively. ANNOVAR (http://annovar.openbioinformatics.org/) was used to annotate the detected variants. Any single nucleotide variant present at $>0.1 \%$ in the Exome Variant Server (https:// evs.gs.washington.edu/) or Single Nucleotide Polymorphism Database was filtered. The variants present in the Catalogue of Somatic Mutations in Cancer (https://cancer.sanger.ac.uk/) were reviewed.

\section{Results}

Clinicopathological characteristics. Table II summarizes the clinicopathological characteristics of 11 patients with HGSC showing wild-type p53 immunostaining pattern. Patient age at initial diagnosis ranged between 51 and 79 years (mean=63.7 years; median=61 years). Histological grade was 3 in eight $(72.7 \%)$ patients and 2 in three $(27.3 \%)$ patients. 
Table I. Gene list.

\begin{tabular}{|c|c|c|c|c|c|c|c|c|}
\hline$A B L 1$ & $B R C A 2$ & $C D K N 1 B$ & $E R G$ & $G N A Q$ & MAP $3 K 4$ & $N F 2$ & PIK3R2 & SMARCA4 \\
\hline$A B L 2$ & BRD2 & $C D K N 2 A$ & ERRFII & GNAS & $M A P K 1$ & NFKBIA & POLE & $S M A R C B 1$ \\
\hline$A K T 1$ & $B R D 3$ & $C D K N 2 B$ & ESR1 & HDAC9 & $M A P K 3$ & $N K X 2-1$ & PPARG & $S M O$ \\
\hline$A K T 2$ & BRD4 & $C D K N 2 C$ & ETV1 & $H G F$ & $M A P K 8$ & NOTCH1 & PTCH1 & $S R C$ \\
\hline$A K T 3$ & $B R A F$ & CEBPA & ETV4 & $H R A S$ & $M C L 1$ & NOTCH2 & PTEN & STK11 \\
\hline$A L K$ & $B R C A 1$ & CHEK2 & ETV5 & $I D H I$ & MDM2 & NOTCH3 & $R A B 35$ & $S Y K$ \\
\hline$A P C$ & $B R C A 2$ & $C R E B B P$ & ETV6 & $I D H 2$ & MDM4 & NOTCH4 & $R A D 50$ & $T E R T$ \\
\hline$A R$ & $B R D 2$ & $C R K L$ & EWSR1 & $I G F 1 R$ & $M E D 12$ & $N P M 1$ & $R A F 1$ & TET2 \\
\hline$A R A F$ & $B R D 3$ & $C S F 1 R$ & $E Z H 2$ & $I G F 2$ & MEN1 & $N R A S$ & $R A R A$ & TMPRSS2 \\
\hline$A S X L 1$ & BRD4 & CTNNB1 & $F B X W 7$ & $J A K 1$ & $M E T$ & NTRK1 & $R B 1$ & TOP $2 A$ \\
\hline$A T M$ & $C B F B$ & $D D R 1$ & FGFR1 & $J A K 2$ & MITF & NTRK2 & $R E T$ & TP53 \\
\hline$A T R$ & $C C N D 1$ & $D D R 2$ & $F G F R 2$ & $J A K 3$ & MLH1 & NTRK3 & RHEB & $T S C 1$ \\
\hline AURKA & CCND2 & DNMT3A & FGFR3 & $K D R$ & $M P L$ & NUTM1 & RICTOR & TSC2 \\
\hline$A U R K B$ & $C C N D 3$ & DOT1L & FGFR4 & KIT & MSH2 & $P D G F B$ & $R N F 43$ & $V H L$ \\
\hline AURKC & CCNE1 & $E G F R$ & $F L C N$ & $K M T 2 A$ & MSH6 & PDGFRA & ROS1 & $W T 1$ \\
\hline$A X L$ & $\mathrm{CDHI}$ & EPHA3 & FLT1 & $K R A S$ & MTOR & $P D G F R B$ & RSPO1 & XPO1 \\
\hline$B A P 1$ & CDK12 & $E R B B 2$ & FLT3 & $M A P 2 K 1$ & $M Y C$ & PIK $3 C A$ & RSPO2 & ZNRF3 \\
\hline$B C L 2$ & $C D K 4$ & ERBB3 & FLT4 & $M A P 2 K 2$ & $M Y C N$ & $P I K 3 C B$ & $R U N X 1$ & \\
\hline$B R A F$ & CDK6 & ERBB4 & FOXL2 & $M A P 2 K 4$ & MYD 88 & PIK3CD & $S M A D 2$ & \\
\hline BRCA1 & $C D K N 1 A$ & $E R C C 2$ & GNA11 & $M A P 3 K 1$ & $N F 1$ & PIK3R1 & SMAD4 & \\
\hline
\end{tabular}

Six (54.5\%) patients presented at FIGO stage IIIC and 3 $(27.3 \%)$ at FIGO stage IVB. The remaining two patients presented at FIGO stage IC $(1 / 11 ; 9.1 \%)$ and IIB $(1 / 11$; $9.1 \%)$, respectively. All $(11 / 11 ; 100.0 \%)$ patients underwent postoperative platinum-based adjuvant chemotherapy. Paired tumor tissue samples were obtained before and after chemotherapy in two (18.2\%) patients; pre-chemotherapeutic samples (samples 8-1 and 9-1) were taken from the tumor tissues obtained with primary debulking surgery (PDS), and post-chemotherapeutic samples (samples 8-2 and 9-2) were taken from the recurrent tumor tissues obtained with secondary debulking surgery (SDS). Two (18.2\%) patients underwent platinum-based neoadjuvant chemotherapy followed by interval debulking surgery (IDS). Similarly, paired samples were obtained before and after chemotherapy in two patients; pre-chemotherapeutic samples (sample 10-1 and 11-1) were taken from biopsy specimens obtained with diagnostic laparoscopy, and post-chemotherapeutic samples (sample 10-2 and 11-2) were taken from the tumor tissues obtained with IDS. Eighteen tissue samples were obtained from the following sites: the right ovary $(6 / 18 ; 33.3 \%)$, left ovary $(2 / 18 ; 11.1 \%)$, left fallopian tube $(2 / 18 ; 11.1 \%)$, pelvic peritoneum $(4 / 18 ; 22.2 \%)$, and omentum $(4 / 18 ; 22.2 \%)$.

All cases showed characteristic histopathological features of HGSC, represented by destructive infiltration of tumor cells forming branching papillary fronds, slit-like fenestrations, and complex glandular architecture (Figure 1A). The tumor cells showed severe nuclear atypia, frequent mitoses, and atypical mitotic figures (Figure 1B). Immunostaining for p53 was performed on 18 tissue samples. In $11 \mathrm{HGSC}$ and one normal fallopian tube sample, p53 expression was patchy and showed weak-to-moderate intensity in the tumor cell nuclei, similar to the wild-type pattern (Figure 1C). In the remaining two HGSC samples, diffuse and strong nuclear p53 immunoreactivity (missense mutation pattern; Figure 1D) and complete absence of p53 expression (nonsense mutation pattern; Figure 1E) were observed, respectively.

NGS results. Table III summarizes the targeted sequencing results of 18 tissue samples, including 15 HGSC samples with wild-type p53 immunostaining pattern, two HGSC samples with mutant p53 immunostaining pattern, and one fallopian tube sample, showing wild-type staining pattern. TP53 mutations were identified in all $(17 / 17 ; 100.0 \%)$ HGSC tissue samples, but not in the normal fallopian tube sample. Six (33.3\%) samples showed a nonsense mutation of the TP53 gene (samples 3, 4, 5, 6, 10-1, and 10-2). Missense TP53 mutations occurred in one (5.6\%) sample (sample 7). Four (22.2\%) samples had frameshift mutations, two of which were frameshift deletions (samples 9-1 and 92 ) and two insertions (samples 11-1 and 11-2). Splice site mutations were identified in four $(22.2 \%)$ samples (samples $1,2,8-1$, and 8-2). Arginine 342 (R342) was the most frequently mutated amino acid, with $40.0 \%(6 / 15)$ of mutations (p.R342* and p.R342fs*3) occurring in this codon.

Five of the nine PDS samples exhibited nonsense mutations (samples 3, 4, 5, 6, and 10-1). Splice site deletions (samples 1,2, and 8-1) and missense mutations (sample 7) were observed in three and one PDS samples, respectively. All (6/15) the remaining samples obtained by SDS, IDS, or laparoscopic biopsy also showed TP53 mutations. In two 


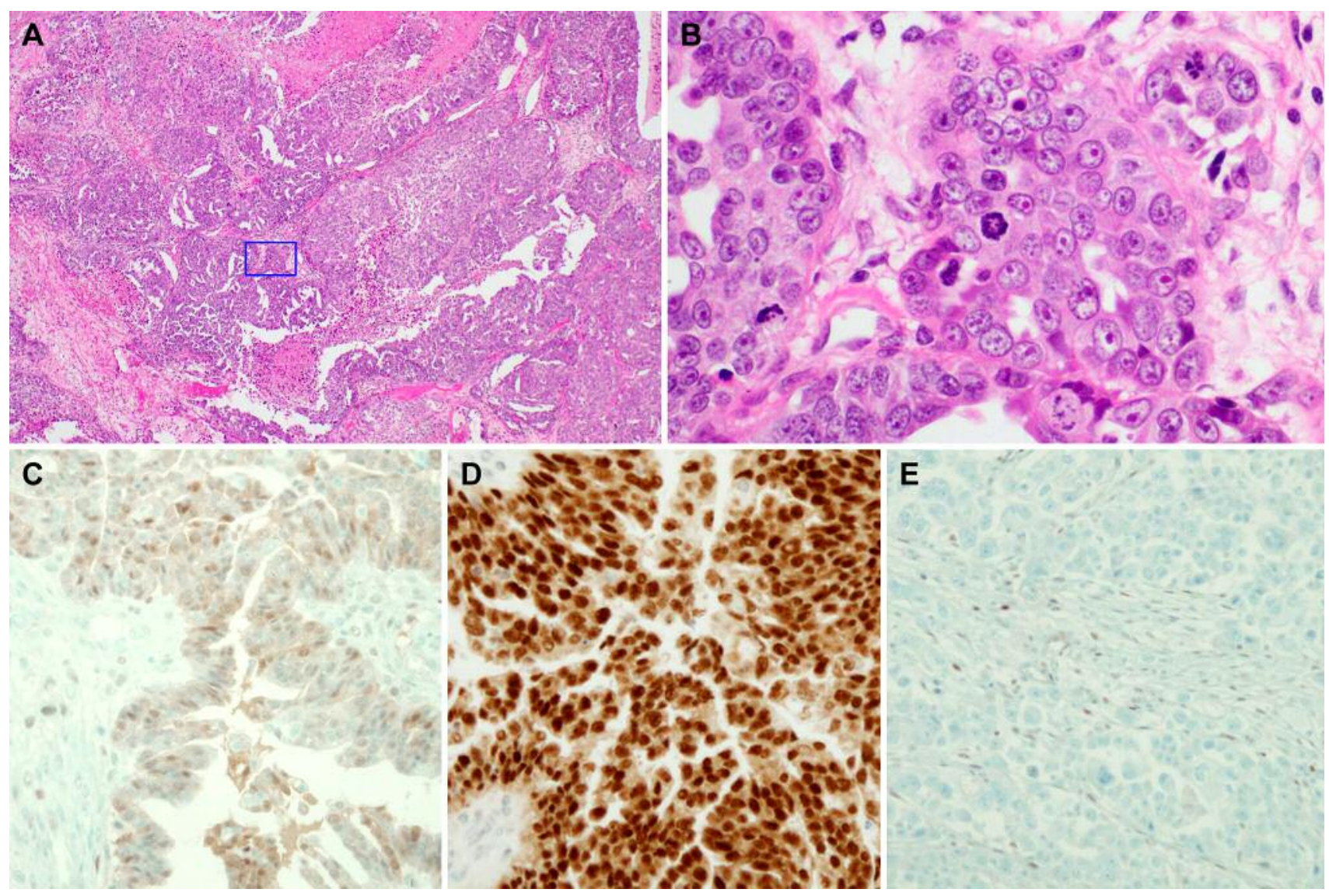

Figure 1. Histopathological and immunohistochemical findings of high-grade serous carcinoma. A: Diffuse growth of tumor cells displaying destructive stromal infiltration, solid and papillary architecture, slit-like fenestrations, and complex glands with intraluminal necrotic debris. B: Higher magnification of the area highlighted in A (blue box) reveals tumor cells showing severe nuclear pleomorphism, conspicuous nucleoli, frequent mitoses, and atypical mitotic figures. C: Patchy and weak p53 expression (wild-type pattern). D: Diffuse and strong p53 expression (missense mutation pattern). E: Complete absence of p53 expression (nonsense mutation pattern). A: Original magnification, A, 40x; B, 400x; $C$, $D$, and $E, 200 x$

patients who underwent neoadjuvant and two who underwent postoperative adjuvant chemotherapy, the TP53 mutations identified in samples obtained after chemotherapy were the same as those detected in pre-chemotherapeutic samples. Two patients had frameshift mutations and the other two had a splice site deletion and a nonsense mutation, respectively. Other mutations classified as variants of uncertain significance are summarized in Table IV (38). The location of the TP53 mutation did not influence tumor location, histological grade, or FIGO stage.

\section{Discussion}

Mutation of TP53 is pervasive and characteristic in tuboovarian and peritoneal HGSC. p53 has been used as a surrogate marker for the presence of TP53 mutations in HGSC (5). When TP53 is mutated, an aberrant immuno- expression pattern is seen in more than $95 \%$ of cases (39). Although a certain number of HGSC cases show wild-type pattern of p53 immunostaining, there have been a few studies showing that, when sequenced, all HGSC cases examined actually bear TP53 mutations $(9,15)$. Considering that p53 immunopositivity is determined by an immunohistochemical staining pattern that correlates with TP53 mutation as opposed to simply a positive or negative staining and that some HGSC cases with truncating or splice site TP53 mutations can show a non-functional p53 expression pattern, the fact that p53 immunostaining cannot accurately predict TP53 mutational status is understandable $(40,41)$.

There are two main chemotherapeutic treatment methods for managing ovarian carcinomas, including HGSC: 1) PDS followed by postoperative platinum-based adjuvant chemotherapy and 2) neoadjuvant chemotherapy followed by IDS. The morphology of post-chemotherapeutic carcinoma is 
Kim et al: Targeted Sequencing of High-grade Serous Carcinoma With Wild-type p53

Table II. Clinicopathological characteristics of patients with HGSC showing wild-type p53 immunostaining pattern.

\begin{tabular}{|c|c|c|c|c|c|}
\hline Sample no & Age (years) & Location & Diagnosis & Histological grade & FIGO stage \\
\hline 1 & 72 & Right ovary & HGSC & 3 & IIIC \\
\hline 2 & 79 & Omentum & HGSC & 2 & IVB \\
\hline 3 & 67 & Posterior $c u l-d e-s a c$ & HGSC & 3 & IIIC \\
\hline 4 & 57 & Left ovary & HGSC & 3 & IIB \\
\hline 5 & 51 & Right ovary & HGSC & 3 & IC \\
\hline 6 & 53 & Posterior cul-de-sac & HGSC & 2 & IIIC \\
\hline 7 & 73 & Omentum & HGSC & 3 & IVB \\
\hline $8-1$ & 52 & Omentum & HGSC & 2 & IIIC \\
\hline $8-2$ & & Pelvic peritoneum & HGSC & & \\
\hline $9-1$ & 71 & Right ovary & HGSC & 3 & IIIC \\
\hline $9-2$ & & Pelvic peritoneum & HGSC & & \\
\hline $10-1$ & 61 & Left ovary & HGSC & 3 & IIIC \\
\hline $10-2$ & & Left fallopian tube & HGSC & & \\
\hline $11-1$ & 65 & Omentum & HGSC & 3 & IVB \\
\hline $11-2$ & & Right ovary & HGSC & & \\
\hline 12 & 59 & Right ovary & HGSC & 3 & IIIC \\
\hline 13 & 45 & Right ovary & HGSC & 3 & IIIC \\
\hline 14 & 42 & Left fallopian tube & Normal & NA & NA \\
\hline
\end{tabular}

FIGO: International Federation of Gynecology and Obstetrics; HGSC: high-grade serous carcinoma; NA: not applicable.

Table III. Targeted sequencing results: Tumor protein 53 (TP53) mutations.

\begin{tabular}{|c|c|c|c|c|c|c|c|c|}
\hline $\begin{array}{l}\text { Sample } \\
\text { no. }\end{array}$ & $\begin{array}{l}\text { Sample } \\
\text { type }\end{array}$ & NAC & POAC & $\begin{array}{l}\text { p53 IHC } \\
\text { pattern }\end{array}$ & $\begin{array}{l}\text { Mutation } \\
\text { type }\end{array}$ & $\begin{array}{l}\text { Sequence } \\
\text { change }\end{array}$ & $\begin{array}{c}\text { Predicted } \\
\text { effect }\end{array}$ & $\begin{array}{c}\text { Clinical } \\
\text { effect }\end{array}$ \\
\hline 1 & PDS & No & No & Wild-type & Splice acceptor variant & c.920-2delA & Splice site deletion & Pathogenic \\
\hline 2 & PDS & No & No & Wild-type & Splice acceptor variant & $\begin{array}{l}\text { c.920-5_923del } \\
\text { CCTAGCACT }\end{array}$ & Splice site deletion & Pathogenic \\
\hline 3 & PDS & No & No & Wild-type & Nonsense variant & c. $1024 \mathrm{C}>\mathrm{T}$ & p.R342* & Pathogenic \\
\hline 4 & PDS & No & No & Wild-type & Nonsense variant & c. $916 \mathrm{C}>\mathrm{T}$ & p.R306* & Pathogenic \\
\hline 5 & PDS & No & No & Wild-type & Nonsense variant & c. $892 \mathrm{G}>\mathrm{T}$ & p.E298* & Pathogenic \\
\hline 6 & PDS & No & No & Wild-type & Nonsense variant & c. $1024 \mathrm{C}>\mathrm{T}$ & p.R342* & Pathogenic \\
\hline 7 & PDS & No & No & Wild-type & Missense variant & c. $488 \mathrm{~A}>\mathrm{G}$ & p.Y163C & Pathogenic \\
\hline $8-1$ & PDS & No & No & Wild-type & Splice acceptor variant & c. $920-1 \mathrm{G}>\mathrm{T}$ & Splice site deletion & Pathogenic \\
\hline $8-2$ & SDS & No & Yes & Wild-type & Splice acceptor variant & c. $920-1 \mathrm{G}>\mathrm{T}$ & Splice site deletion & Pathogenic \\
\hline $9-1$ & PDS & No & No & Wild-type & Frameshift variant & c.1024delC & p.R342fs*3 & Pathogenic \\
\hline $9-2$ & SDS & No & Yes & Wild-type & Frameshift variant & c.1024delC & p.R342fs*3 & Pathogenic \\
\hline $10-1$ & Biopsy & No & No & Wild-type & Nonsense variant & c. $1024 \mathrm{C}>\mathrm{T}$ & p.R342* & Pathogenic \\
\hline $10-2$ & IDS & Yes & No & Wild-type & Nonsense variant & c. $1024 \mathrm{C}>\mathrm{T}$ & p.R342* & Pathogenic \\
\hline $11-1$ & Biopsy & No & No & Wild-type & Frameshift variant & c.864_865insTT & p.L289fs*23 & Pathogenic \\
\hline $11-2$ & IDS & Yes & No & Wild-type & Frameshift variant & c.864_865insTT & p.L289fs $* 23$ & Pathogenic \\
\hline $12(\mathrm{PC})$ & PDS & No & No & Missense & Missense variant & c. $743 \mathrm{G}>\mathrm{A}$ & p.R248Q & Pathogenic \\
\hline $13(\mathrm{PC})$ & PDS & No & No & Nonsense & Nonsense variant & c. $586 \mathrm{C}>\mathrm{T}$ & p.R196* & Pathogenic \\
\hline $14(\mathrm{NC})$ & LSO & No & No & Wild-type & Wild-type & NA & NA & NA \\
\hline
\end{tabular}

IDS: Interval debulking surgery; IHC: immunohistochemical staining; LSO: left salpingo-oophorectomy; NA: not applicable; NAC: Neoadjuvant chemotherapy; NC: negative control; PC: positive control; PDS: primary debulking surgery; POAC: postoperative adjuvant chemotherapy; SDS: secondary debulking surgery.

different from its pretreatment appearance, making histological subtyping and grading difficult (40). Meanwhile, despite the tremendous genomic diversity that develops at very early stages of carcinogenesis (42), ancestral clones persist, irrespective of disease progression and chemotherapeutic intervention (43). In this study, we confirmed the presence of TP53 mutations in HGSC showing a wild-type p53 immunostaining pattern and demonstrated the mutational 
Table IV. Targeted sequencing results: Variants of uncertain significance.

\begin{tabular}{|c|c|c|c|c|}
\hline Sample No & Gene & Sequence change & Predicted effect & Clinical effect \\
\hline 2 & $J A K 2$ & c. $2958 \mathrm{C}>\mathrm{T}$ & p.N986N & Uncertain significance \\
\hline 3 & ATR & c. $2688 \mathrm{G}>\mathrm{A}$ & p.L896L & Uncertain significance \\
\hline \multirow[t]{2}{*}{4} & $R A D 50$ & c. $1831 \mathrm{~A}>\mathrm{G}$ & p.I611V & Uncertain significance \\
\hline & STK11 & c. $* 201 \mathrm{G}>\mathrm{A}$ & 3'-UTR variant & Uncertain significance \\
\hline \multirow[t]{2}{*}{$8-1$} & $A T R$ & c.5899-9delT & Splice site variant & Uncertain significance \\
\hline & NOTCH1 & c. $3970 \mathrm{G}>\mathrm{A}$ & p.V1324M & Uncertain significance \\
\hline \multirow[t]{4}{*}{$8-2$} & ATR & c.5899-9delT & Splice site variant & Uncertain significance \\
\hline & NOTCH1 & c. $3970 \mathrm{G}>\mathrm{A}$ & p.V1324M & Uncertain significance \\
\hline & $N F 1$ & c. $3871-4 \mathrm{G}>\mathrm{A}$ & Splice site variant & Uncertain significance \\
\hline & $N F 1$ & c. $4526 \mathrm{G}>\mathrm{A}$ & p.R1509H & Uncertain significance \\
\hline $9-1$ & $M E D 12$ & c.6256_6258delCAG & p.Q2086del & Uncertain significance \\
\hline $9-2$ & MED12 & c.6256_6258delCAG & p.Q2086del & Uncertain significance \\
\hline \multirow[t]{2}{*}{$10-1$} & ATR & c.5899-9delT & Splice site variant & Uncertain significance \\
\hline & $M E D 12$ & c.6256_6258delCAG & p.Q2086del & Uncertain significance \\
\hline \multirow[t]{2}{*}{$10-2$} & $A T R$ & c.5899-9delT & Splice site variant & Uncertain significance \\
\hline & MED12 & c.6256_6258delCAG & p.Q2086del & Uncertain significance \\
\hline
\end{tabular}

constancy between matched pre- and post-chemotherapeutic HGSC tissue samples. This result is not only concordant with a previous study reporting pre- and post-chemotherapeutic p53 expression in HGSC using only immunohistochemistry (40), but also presents confirmatory evidence via NGS. Our data suggest that similar p53 immunoexpression patterns in preand post-chemotherapeutic samples indicate the presence of the same TP53 mutation.

As per our observations, even if an HGSC case presents wild-type p53 immunostaining pattern, there is still a possibility that the tumor bears a TP53 mutation, which could be a non-typical mutation, such as a splice site mutation or frameshift deletion. Furthermore, if an HGSC case bearing a TP53 mutation shows the presence of the mutation before and after chemotherapy, pathologists can make a definite diagnosis of HGSC using NGS targeting TP53 even when the tumor displays wild-type p53 immunostaining pattern or when there are significant morphological alterations induced by chemotherapy. Our results support the notion that TP53 mutations are invariably present in cases of tubo-ovarian and peritoneal HGSC, and that the TP53 mutation persists even after chemotherapy. In HGSC cases with a wild-type p53 immunostaining pattern or with disseminated metastatic lesions of unclear origin after chemotherapy, targeted sequencing using NGS technique can be helpful in revealing the presence of the TP53 mutation.

In conclusion, we observed that all HGSC cases examined exhibited TP53 mutations, confirming the fact that all HGSCs are TP53-mutants even though a subset of HGSCs display a wild-type p53 immunostaining pattern. We did not observe any significant difference in the type of TP53 mutation between paired specimens obtained before and after chemotherapy, suggesting that chemotherapy does not affect TP53 mutational status in tubo-ovarian and peritoneal HGSC.

\section{Conflicts of Interest}

None of the Authors have any conflicts of interest to declare regarding this study.

\section{Authors' Contributions}

All Authors were responsible for substantial contributions to the conception and design of the study, acquisition of data, analysis and interpretation of the data, as well as drafting the manuscript, revising the manuscript critically for important intellectual content, and providing final approval of the version to be published.

\section{Acknowledgements}

This research was supported by the National Research Foundation of Korea (NRF) grant funded by the Korean government (MSIT) (2018R1C1B5043725) and the Basic Science Research Program through the NRF funded by the Ministry of Education (2016R1D1A1B03935584).

\section{References}

1 Kastenhuber ER and Lowe SW: Putting p53 in context. Cell 170: 1062-1078, 2017. PMID: 28886379. DOI: 10.1016/ j.cell.2017.08.028

2 Ungerleider NA, Rao SG, Shahbandi A, Yee D, Niu T, Frey WD and Jackson JG: Breast cancer survival predicted by TP53 mutation status differs markedly depending on treatment. Breast Cancer Res 20: 115, 2018. PMID: 30285883. DOI: 10.1186/ s13058-018-1044-5 
3 Aubrey BJ, Strasser A and Kelly GL: Tumor-suppressor functions of the TP53 pathway. Cold Spring Harb Perspect Med 6, 2016. PMID: 27141080. DOI: 10.1101/cshperspect.a026062

4 Brady CA and Attardi LD: p53 at a glance. J Cell Sci 123: 25272532, 2010. PMID: 20940128. DOI: 10.1242/jcs.064501

5 Cole AJ, Dwight T, Gill AJ, Dickson KA, Zhu Y, Clarkson A, Gard GB, Maidens J, Valmadre S, Clifton-Bligh R and Marsh DJ: Assessing mutant p53 in primary high-grade serous ovarian cancer using immunohistochemistry and massively parallel sequencing. Sci Rep 6: 26191, 2016. PMID: 27189670. DOI: 10.1038/srep26191

6 Rogel A, Popliker M, Webb CG and Oren M: p53 cellular tumor antigen: Analysis of mRNA levels in normal adult tissues, embryos, and tumors. Mol Cell Biol 5: 2851-2855, 1985. PMID: 3915536.

7 Yemelyanova A, Vang R, Kshirsagar M, Lu D, Marks MA, Shih Ie $M$ and Kurman RJ: Immunohistochemical staining patterns of p53 can serve as a surrogate marker for TP53 mutations in ovarian carcinoma: An immunohistochemical and nucleotide sequencing analysis. Mod Pathol 24: 1248-1253, 2011. PMID: 21552211. DOI: $10.1038 / \bmod$ pathol.2011.85

8 Bennett WP, Hollstein MC, Hsu IC, Sidransky D, Lane DP, Vogelstein B and Harris CC: Mutational spectra and immunohistochemical analyses of p53 in human cancers. Chest 101: 19S-20S, 1992. PMID: 1541189.

9 Na K, Sung JY and Kim HS: TP53 mutation status of tuboovarian and peritoneal high-grade serous carcinoma with a wildtype p53 immunostaining pattern. Anticancer Res 37: 66976703, 2017. PMID: 29187446. DOI: 10.21873 /anticanres.12128

10 Lax SF, Kendall B, Tashiro H, Slebos RJ and Hedrick L: The frequency of $p 53, K$-ras mutations, and microsatellite instability differs in uterine endometrioid and serous carcinoma: Evidence of distinct molecular genetic pathways. Cancer 88: 814-824, 2000. PMID: 10679651.

11 Shahin MS, Hughes JH, Sood AK and Buller RE: The prognostic significance of $p 53$ tumor-suppressor gene alterations in ovarian carcinoma. Cancer 89: 2006-2017, 2000. PMID: 11064359.

12 Tashiro H, Isacson C, Levine R, Kurman RJ, Cho KR and Hedrick L: p53 gene mutations are common in uterine serous carcinoma and occur early in their pathogenesis. Am J Pathol 150: 177-185, 1997. PMID: 9006334.

13 Bray F, Ferlay J, Soerjomataram I, Siegel RL, Torre LA and Jemal A: Global cancer statistics 2018: GLOBOCAN estimates of incidence and mortality worldwide for 36 cancers in 185 countries. CA Cancer J Clin 68: 394-424, 2018. PMID: 30207593. DOI: $10.3322 /$ caac. 21492

14 Visintin I, Feng Z, Longton G, Ward DC, Alvero AB, Lai Y, Tenthorey J, Leiser A, Flores-Saaib R, Yu H, Azori M, Rutherford T, Schwartz PE and Mor G: Diagnostic markers for early detection of ovarian cancer. Clin Cancer Res 14: 10651072, 2008. PMID: 18258665. DOI: 10.1158/1078-0432.CCR07-1569

15 Vang R, Levine DA, Soslow RA, Zaloudek C, Shih Ie M and Kurman RJ: Molecular alterations of TP53 are a defining feature of ovarian high-grade serous carcinoma: A re-review of cases lacking TP53 mutations in The Cancer Genome Atlas ovarian study. Int J Gynecol Pathol 35: 48-55, 2016. PMID: 26166714. DOI: $10.1097 /$ PGP.0000000000000207

16 Hall J, Paul J and Brown R: Critical evaluation of p53 as a prognostic marker in ovarian cancer. Expert Rev Mol Med 6: 120, 2004. PMID: 15147608. DOI: 10.1017/S1462399404007781
17 Wojnarowicz PM, Oros KK, Quinn MC, Arcand SL, Gambaro K, Madore J, Birch AH, de Ladurantaye M, Rahimi K, Provencher DM, Mes-Masson AM, Greenwood CM and Tonin PN: The genomic landscape of TP53 and p53 annotated high grade ovarian serous carcinomas from a defined founder population associated with patient outcome. PLoS One 7: e45484, 2012. PMID: 23029043. DOI: 10.1371/journal.pone.0045484

18 Na K, Sung JY and Kim HS: Clinicopathological characteristics of high-grade squamous intraepithelial lesions involving condyloma acuminatum. Anticancer Res 38: 1767-1774, 2018. PMID: 29491115. DOI: 10.21873/anticanres.12414

$19 \mathrm{Na} \mathrm{K}$ and Kim HS: Clinicopathological characteristics of fallopian tube metastases from primary endometrial, cervical, and nongynecological malignancies: A single institutional experience. Virchows Arch 471: 363-373, 2017. PMID: 28702779. DOI: $10.1007 / \mathrm{s} 00428-017-2186-\mathrm{z}$

$20 \mathrm{Na} \mathrm{K}$, Kim EK, Jang W and Kim HS: CTNNB1 mutations in ovarian microcystic stromal tumors: Identification of a novel deletion mutation and the use of pyrosequencing to identify reported point mutation. Anticancer Res 37: 3249-3258, 2017. PMID: 28551672. DOI: 10.21873/anticanres.11688

$21 \mathrm{Na}$ K, Park SY and Kim HS: Clinicopathological characteristics of primary ovarian adenomyoma: A single-institutional experience. Anticancer Res 37: 2565-2574, 2017. PMID: 28476828. DOI: 10.21873 /anticanres. 11600

$22 \mathrm{Na} \mathrm{K}$, Sung JY and Kim HS: Stromal p16 overexpression in adult granulosa cell tumors of the ovary. Anticancer Res 37: 2437-2444, 2017. PMID: 28476811. DOI: 10.21873/anticanres. 11583

$23 \mathrm{Na} \mathrm{K}$ and Kim HS: Clinicopathologic and molecular characteristics of mesonephric adenocarcinoma arising from the uterine body. Am J Surg Pathol 43: 12-25, 2019. PMID: 29189288. DOI: 10.1097/PAS.0000000000000991

$24 \mathrm{Na} \mathrm{K}$, Lee JY, Sung JY, Kim GM, Koo JS and Kim HS: Comparative clinicopathological and cytomorphological analyses of peritoneal carcinomatosis associated with metastatic breast carcinoma and primary peritoneal/ovarian carcinoma in patients with a history of breast carcinoma. Virchows Arch 473: 165-175, 2018. PMID: 29926183. DOI: 10.1007/s00428-018-2390-5

25 Park CK and Kim HS: Clinicopathological characteristics of ovarian metastasis from colorectal and pancreatobiliary carcinomas mimicking primary ovarian mucinous tumor. Anticancer Res 38: 5465-5473, 2018. PMID: 30194204. DOI: 10.21873/anticanres.12879

26 Chung T, Do SI, Na K, Kim G, Jeong YI, Kim YW and Kim HS: Stromal p16 overexpression in gastric-type mucinous carcinoma of the uterine cervix. Anticancer Res 38: 3551-3558, 2018. PMID: 29848709. DOI: 10.21873/anticanres.12627

27 Joo JW, Kim HS, Do SI and Sung JY: Expression of zinc finger and BTB domain-containing 7A in colorectal carcinoma. Anticancer Res 38: 2787-2792, 2018. PMID: 29715100. DOI: 10.21873/anticanres.12522

28 Sung JY, Jung YY and Kim HS: Clinicopathological characteristics and KRAS mutation status of endometrial mucinous metaplasia and carcinoma. Anticancer Res 38: 27792786, 2018. PMID: 29715099. DOI: 10.21873/anticanres.12521

29 Jung YY, Sung JY, Kim JY and Kim HS: Down-regulation of Bcell translocation gene 1 by promoter methylation in colorectal carcinoma. Anticancer Res 38: 691-697, 2018. PMID: 29374692. DOI: 10.21873 /anticanres.12274 
30 Kim JY, Na K and Kim HS: Clinicopathological characteristics of mitotically-active cellular fibroma of the ovary: A singleinstitutional experience. Anticancer Res 37: 2557-2564, 2017. PMID: 28476827. DOI: 10.21873/anticanres.11599

31 Jang M, Sung JY, Kim JY and Kim HS: Clinicopathological characteristics of metaplastic papillary tumor of the fallopian tube. Anticancer Res 37: 3693-3701, 2017. PMID: 28668862.

32 Do SI, Yoon G, Kim HS, Kim K, Lee H, Do IG, Kim DH, Chae SW and Sohn JH: Increased brahma-related gene 1 expression predicts distant metastasis and shorter survival in patients with invasive ductal carcinoma of the breast. Anticancer Res 36: 4873 4882, 2016. PMID: 27630343. DOI: 10.21873/anticanres.11051

33 Bae GE, Yoon N, Cho EY, Kim HS and Cho SY: Clinicopathological and molecular characteristics of mammary adenoid cystic carcinoma with adipocytic differentiation with emphasis on the identification of a novel BRAF mutation. Anticancer Res 39: 369-374, 2019. PMID: 30591482. DOI: 10.21873/ anticanres.13121

34 Bae GE, Do SI, Kim K, Park JH, Cho S and Kim HS: Increased sphingosine kinase 1 expression predicts distant metastasis and poor outcome in patients with colorectal cancer. Anticancer Res 39: 663-670, 2019. PMID: 30711943. DOI: 10.21873/anticanres. 13161

$35 \mathrm{Kim}$ M and Kim HS: Clinicopathological characteristics of welldifferentiated papillary mesothelioma of the peritoneum: A single-institutional experience of 12 cases. In Vivo 33: 633-642, 2019. PMID: 30804152. DOI: 10.21873/invivo.11521

36 Rhead B, Karolchik D, Kuhn RM, Hinrichs AS, Zweig AS, Fujita PA, Diekhans M, Smith KE, Rosenbloom KR, Raney BJ, Pohl A, Pheasant M, Meyer LR, Learned K, Hsu F, HillmanJackson J, Harte RA, Giardine B, Dreszer TR, Clawson H, Barber GP, Haussler D and Kent WJ: The UCSC Genome Browser database: Update 2010. Nucleic Acids Res 38: D613619, 2010. PMID: 19906737. DOI: 10.1093/nar/gkp939

37 Mills RE, Luttig CT, Larkins CE, Beauchamp A, Tsui C, Pittard WS and Devine SE: An initial map of insertion and deletion (INDEL) variation in the human genome. Genome Res 16: 11821190, 2006. PMID: 16902084. DOI: 10.1101/gr.4565806

38 Li MM, Datto M, Duncavage EJ, Kulkarni S, Lindeman NI, Roy S, Tsimberidou AM, Vnencak-Jones CL, Wolff DJ, Younes A and Nikiforova MN: Standards and Guidelines for the Interpretation and Reporting of Sequence Variants in Cancer: A Joint Consensus Recommendation of the Association for Molecular Pathology, American Society of Clinical Oncology, and College of American Pathologists. J Mol Diagn 19: 4-23, 2017. PMID: 27993330. DOI: 10.1016/j.jmoldx.2016.10.002
39 Kobel M, Piskorz AM, Lee S, Lui S, LePage C, Marass F, Rosenfeld N, Mes Masson AM and Brenton JD: Optimized p53 immunohistochemistry is an accurate predictor of TP53 mutation in ovarian carcinoma. J Pathol Clin Res 2: 247-258, 2016. PMID: 27840695. DOI: 10.1002/cjp2.53

40 Casey L, Köbel M, Ganesan R, Tam S, Prasad R, Böhm S, Lockley M, Jeyarajah AJ, Brockbank E, Faruqi A, Gilks CB and Singh N: A comparison of p53 and WT1 immunohistochemical expression patterns in tubo-ovarian high-grade serous carcinoma before and after neoadjuvant chemotherapy. Histopathology 71 : 736-742, 2017. PMID: 28570008. DOI: 10.1111/his.13272

41 Vang R, Shih I-M and Kurman RJ: Ovarian low-grade and highgrade serous carcinoma: pathogenesis, clinicopathologic and molecular biologic features, and diagnostic problems. Adv Anat Pathol 16: 267-282, 2009. PMID: 19700937. DOI: 10.1097/ PAP.0b013e3181b4fffa

42 Bashashati A, Ha G, Tone A, Ding J, Prentice LM, Roth A, Rosner J, Shumansky K, Kalloger S, Senz J, Yang W, McConechy M, Melnyk N, Anglesio M, Luk MT, Tse K, Zeng T, Moore R, Zhao Y, Marra MA, Gilks B, Yip S, Huntsman DG, McAlpine JN and Shah SP: Distinct evolutionary trajectories of primary high-grade serous ovarian cancers revealed through spatial mutational profiling. J Pathol 231: 21-34, 2013. PMID: 23780408. DOI: $10.1002 /$ path.4230

43 Castellarin M, Milne K, Zeng T, Tse K, Mayo M, Zhao Y, Webb JR, Watson PH, Nelson BH and Holt RA: Clonal evolution of high-grade serous ovarian carcinoma from primary to recurrent disease. J Pathol 229: 515-524, 2013. PMID: 22996961. DOI: 10.1002/path.4105
Received June 4, 2019

Revised June 27, 2019

Accepted June 28, 2019 